\title{
A loss-less superconducting kilo-oersted ac magnetic field system
}

\author{
L.J.M. van de Klundert, M.R.E. Bos, and L.C. van der Marel
}

A growing interest has developed in the behaviour of superconductors in alternating fields, mainly due to the possible use of superconducting materials for large scale applications. ${ }^{1}$ Investigations on this topic on a laboratory scale are usually carried out by induction methods. These experiments can be roughly divided into two groups: in the first the samples are subjected to large amplitude alternating fields (up to $2 \mathrm{kOe}$ ), in the second smaller amplitudes biased by a dc field are used. Both methods have their specific merits and disadvantages. In the latter for instance the determination of the time independent component of the average induction inside the sample may be a problem.

So far both liquid nitrogen cooled and superconducting coil systems have been used for generating the applied field. Obviously liquid nitrogen cooled systems require much larger power supplies than the superconducting ones.

For the first type of experiments the field coils can either be driven directly from the power supply ${ }^{2}$ or to overcome the high voltages needed at higher frequencies, by a resonance technique ${ }^{3}$ which will be discussed in the next section in more detail. The second type of experiments can be performed in two ways: by separate coil systems, ${ }^{4}$ each of them generating one of the components $H_{0}$ and $h \cos \omega t$ of the total field or by feeding a modulated current ${ }^{5,6}$ into one coil. In this way the problem of decoupling the two coil systems magnetically can be avoided. But losses may still be present in the coil. ${ }^{5}$

In this paper a description is given of a simple superconducting system which enables us to generate almost loss-less ac magnetic fields in the kilo-oersted region and in the frequency range from dc up to more than $1 \mathrm{kHz}$. The behaviour of various commercially available superconducting wires and the influence of the winding of the coils are also investigated.

\section{General principle}

The design starts from the transformer principle without core (Fig. 1). Both coils are made of superconducting wire; the secondary coil is the field coil. The ac field is generated by means of the primary coil. The field coil can be brought into resonance with the help of an appropriate condensor,

The authors are with the Twente University of Technology, PO Box 217, Enschede, The Netherlands. Received 10 October 1976.

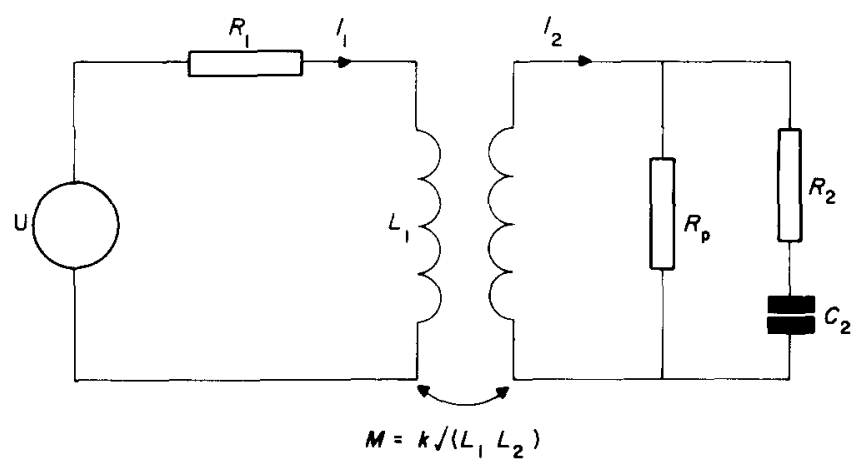

Fig. 1 Plan of the circuit

$C_{2}$, connected in parallel. This condensor, which is placed outside the cryostat, consists of a set of parallel condensors in order to reduce the electrical resistance and in this way the total series resistance in the resonance circuit has been minimized. When the transformer is operated at its resonance frequency only (a small amount of) power has to be supplied in order to compensate for the losses in the secondary circuit, mainly in the condensor, In this way small and cheap power supplies can be used. Evaporation of the helium is mainly due to dissipation in the current leads of the secondary circuit.

\section{Current ratio and resonance frequency}

The current in the field coil is induced by a small primary coil which has been wound around the field coil. In Fig. 1 the fundamental outline of the system is shown. $R_{1}$ represents the total internal resistance of the power supply and a load resistance; $R_{2}$ is the total series resistance of the resonance circuit consisting of three parts: a small resistor $(0.01 \Omega)$ to measure the secondary current $I_{2}$, the series resistance of the condensor, and the resistance of the current leads.

The losses in the field coil have been accounted for by a resistance $R_{\mathrm{p}}$ parallel to the field coil.

To calculate the current ratio $I_{2} / I_{1}$ and the resonance frequency of the field coil the usual calculation technique has been applied; reference can be made to any elementary text book on network analysis. 


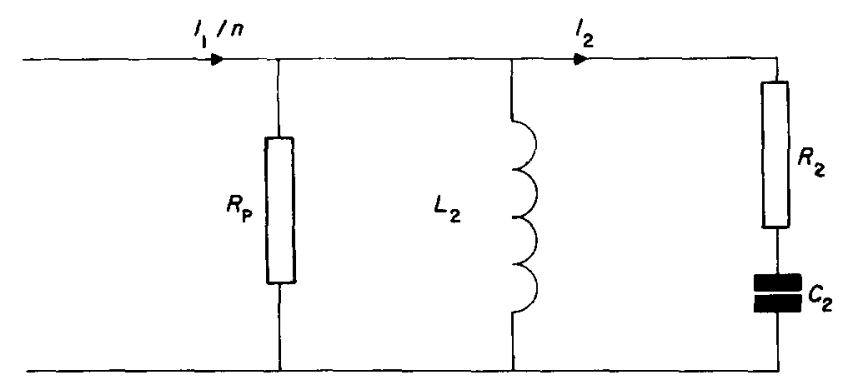

Fig. 2 Transformed plan of the circuit

The essential part of the fundamental scheme of Fig. 1 can be transformed into the one presented in Fig. 2. The primary current $I_{1}$ has been transformed into: $(1 / n) I_{1}$ with $n=(1 / k) \sqrt{ }\left(L_{2} / L_{1}\right) ; L_{2}$ and $L_{1}$ are the self-inductances of the secondary and primary coil respectively and $k$ the coupling coefficient. The frequency dependent current ratio can be written

$$
\frac{I_{2}}{I_{1}}=\frac{1}{n} \frac{R_{\mathrm{p}}}{R_{\mathrm{p}}+R_{2}} \times \frac{\left(\frac{j \omega}{\omega_{0}}\right)^{2}}{1+\frac{j \omega}{\omega_{0}} \frac{1}{Q}+\left(\frac{j \omega}{\omega_{0}}\right)^{2}}
$$

with

$$
\frac{1}{\omega_{0}^{2}}=L_{2} C_{2} \quad\left(1+\frac{R_{2}}{R_{\mathrm{p}}}\right)
$$

and

$$
\frac{1}{Q}=\left(\frac{L_{2}}{R_{p}}+R_{2} C_{2}\right) \omega_{0}
$$

For the resonance frequency, which is the frequency where $\left|I_{2} / I_{1}\right|$ has its maximal value, it is found that

$$
\frac{1}{\omega_{\mathrm{r}}^{2}}=L_{2} C_{2}-\frac{R_{2}^{2} C_{2}^{2}}{2}-\frac{L_{2}^{2}}{2 R_{\mathrm{p}}^{2}}
$$

In practice $R_{\mathrm{p}} \gg R_{2}$ and $\omega_{\mathrm{r}} \sim \omega_{0}$ so we can write

$$
\left|\frac{I_{2}}{I_{1}}\right|_{\max } \sim \frac{Q}{n}
$$

\section{Experimental results}

Dependance of $\left|I_{2} / I_{1}\right|_{\max }$ on $I_{2}$. The first measurements were carried out on a coil system consisting of two secondary and two primary coils wound, on top of each other, on the same coil holder. The diameter of the celeron coil holder was $3 \mathrm{~cm}$. The most important data about the coils are presented in Table 1 .

The first experimental results can be summarized as follows:

(a) $\left|I_{2} / I_{1}\right|_{\max }$ changes linearly with $\sqrt{ } L_{1}$

(b) $\left|I_{2} / I_{1}\right|_{\max }$ is almost independent of $I_{2}$ when the secondary coil consists of one layer

(c) $\left|I_{2} / I_{1}\right|_{\max }$ strongly decreases with increasing $I_{2}$ when the six layer secondary coil is used.

Result (a) can be understood in the following way.

By taking $R_{\mathrm{p}}>R_{2}$ we get

$$
\frac{1}{Q}=\frac{1}{R_{\mathrm{p}}} \sqrt{\frac{L_{2}}{C_{2}}}+R_{2} \sqrt{\frac{C_{2}}{L_{2}}} \text { and with } n=\frac{1}{k} \sqrt{\frac{L_{2}}{L_{1}}}
$$

and so

$$
\left|\frac{I_{2}}{I_{1}}\right|_{\max }=\frac{Q}{n}=\frac{k \sqrt{ } L_{1} C_{2}}{\frac{L_{2}}{R_{\mathrm{p}}}+R_{2} C_{2}}
$$

which denotes that, in accordance with the experimental results $\left|I_{2} / I_{1}\right|_{\max }$ is proportional to $\sqrt{ } L_{1}$.

With regard to the results mentioned in (b) and (c) it may be noted that the inner layers of the six layer coil are subjected to the alternating magnetic field of the outer layers; eddy current losses are generated and so the maximum value of $I_{2}$ will be reduced.

Since the one layer coil has no inner layers, eddy current effects will play a minor role and so $\left|I_{2} / I_{1}\right|_{\max }$ will be independent of $I_{2}$.

\section{Loss reduction; results on coils, made of thin superconducting wire with CuNi matrix}

The Vacuumschmelze $\mathrm{F} 60,0.20$ superconducting wire consists of 60 superconducting filaments of $\mathrm{NbTi}$ in a matrix of copper. Eddy current losses can be reduced by using a matrix material with high electrical resistivity. By applying as few layers as possible for a given number of turns per unit

Table 1

\begin{tabular}{lcccrccc}
\hline Type & No of turns & No of layers & Length & Self inductance & Diameter of wire & Type of wire \\
\hline Primary & 93 & 1 & $5 \mathrm{~cm}$ & 172 & $\mu \mathrm{H}$ & $0.50 \mathrm{~mm}$ & Niomax S25/50 \\
Primary & 186 & 1 & $10 \mathrm{~cm}$ & 360 & $\mu \mathrm{H}$ & $0.50 \mathrm{~mm}$ & Niomax S25/50 \\
Secondary & 415 & 1 & $10 \mathrm{~cm}$ & 2 & $\mathrm{mH}$ & $0.20 \mathrm{~mm}$ & Vacuumschmelze F60, 0.20 \\
Secondary & 2405 & 6 & $10 \mathrm{~cm}$ & 51 & $\mathrm{mH}$ & $0.20 \mathrm{~mm}$ & Vacuumschmelze F60, 0.20 \\
\hline
\end{tabular}


Table 2

\begin{tabular}{|c|c|c|c|c|c|c|c|}
\hline Type & No of turns & No of layers & Length & Diameter of wire & Type of wire & Indi & ted in Fig. 5 \\
\hline Primary & 172 & 1 & $5 \mathrm{~cm}$ & $0.25 \mathrm{~mm}$ & Niomax FM A61/25 & \multirow{3}{*}{$\begin{array}{l}a \\
b\end{array}$} & \multirow{3}{*}{$\begin{array}{l}\text { (at } 4.2 \mathrm{~K} \text { ) } \\
\text { (at } 1.4 \mathrm{~K} \text { ) }\end{array}$} \\
\hline Primary & 344 & 1 & $10 \mathrm{~cm}$ & $0.25 \mathrm{~mm}$ & Niomax FM A61/25 & & \\
\hline Secondary & 1530 & 1 & $10 \mathrm{~cm}$ & $0.05 \mathrm{~mm}$ & Niomax CN A61/05 & & \\
\hline Primary & 171 & 1 & $5 \mathrm{~cm}$ & $0.25 \mathrm{~mm}$ & Niomax FM A61/25 & \multirow{3}{*}{$\begin{array}{l}c \\
d\end{array}$} & \multirow{3}{*}{$\begin{array}{l}\text { (at } 4.2 \mathrm{~K} \text { ) } \\
\text { (at } 1.4 \mathrm{~K} \text { ) }\end{array}$} \\
\hline Primary & 342 & 1 & $10 \mathrm{~cm}$ & $0.25 \mathrm{~mm}$ & Niomax FM A61/25 & & \\
\hline Secondary & 2848 & 2 & $10 \mathrm{~cm}$ & $0.05 \mathrm{~mm}$ & Niomax CN A61/05 & & \\
\hline Primary & 94 & 1 & $5 \mathrm{~cm}$ & $0.50 \mathrm{~mm}$ & Niomax S 25/50 & \multirow{3}{*}{$\begin{array}{l}e \\
f\end{array}$} & \multirow{3}{*}{$\begin{array}{l}\text { (at } 4.2 \mathrm{~K} \text { ) } \\
\text { (at } 1.4 \mathrm{~K})\end{array}$} \\
\hline Primary & 188 & 1 & $10 \mathrm{~cm}$ & $0.50 \mathrm{~mm}$ & Niomax S 25/50 & & \\
\hline Secondary & 5860 & 4 & $10 \mathrm{~cm}$ & $0.05 \mathrm{~mm}$ & Niomax CN 61/05 & & \\
\hline
\end{tabular}

length, the magnetic field that is felt by the superconducting wire, is minimized. On account of these considerations three coil systems have been made in which the secondary coil was wound with a superconducting wire with a diameter of $0.05 \mathrm{~mm}$. This wire consists of 61 filaments in a matrix of $\mathrm{CuNi}$, the resistivity of which is more than a factor of 100 higher than $\mathrm{Cu}$. Table 2 gives more data about these coil systems.

Measurements on these coils showed that the ratio $\left|I_{2} / I_{1}\right|_{\max }$ is almost independent of $I_{2}$ up to a certain value of $I_{2}$ which we denote by $I_{\max }$; at this value of $I_{\max }$ this ratio decreases by a factor of more than a hundred. Obviously the superconducting state has been disturbed at that value of $I_{2}$. In Fig. 3 the values of $I_{\max }$ have been plotted as a function of frequency. From these curves it can be seen that the maximum current through the field coil, $I_{\max }$, has a smaller value when the number of layers of the coil is larger, and, moreover, that better results are obtained at lower temperatures. This is also shown in Fig. 4 where $I_{\max }$ is plotted against temperature at a frequency of $31 \mathrm{~Hz}$ with a field coil, consisting of one layer of 1530 turns. It should be noted that in the low frequency limit $(\nu<10 \mathrm{~Hz})$ the values of $I_{\max }$ are only slightly smaller than the maximum dc currents in the secondary coil.

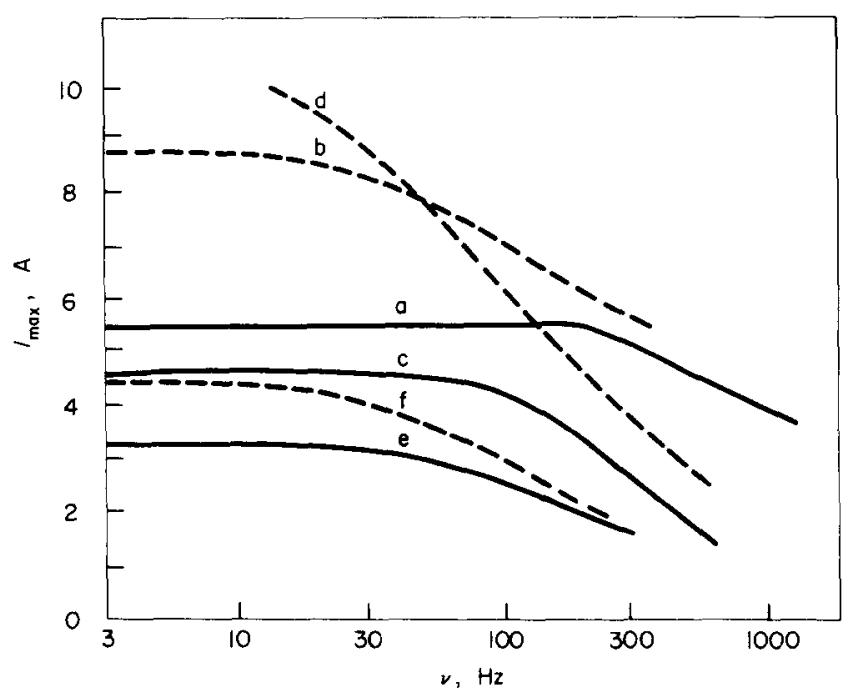

Fig. $3 I_{\max }$ as a function of frequency and temperature for the coils indicated in Table 2

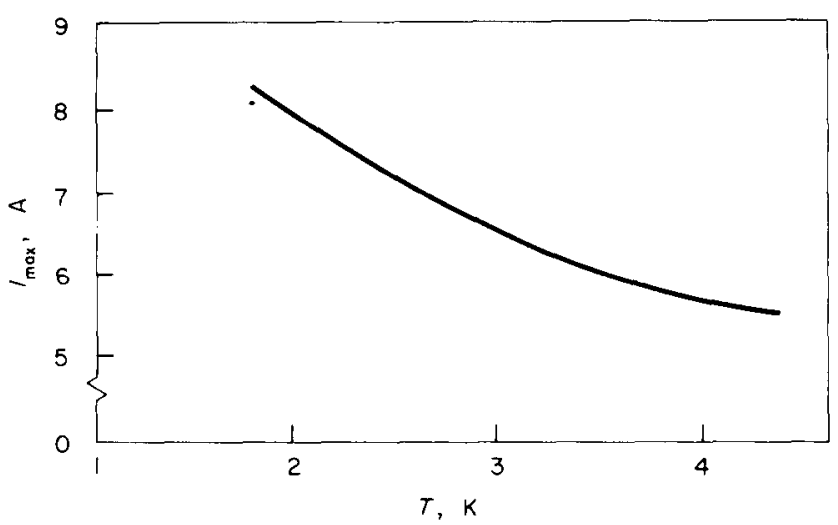

Fig. $4 I_{\max }$ as a function of temperature for the secondary coil with 1530 turns; $\nu=31 \mathrm{~Hz}$

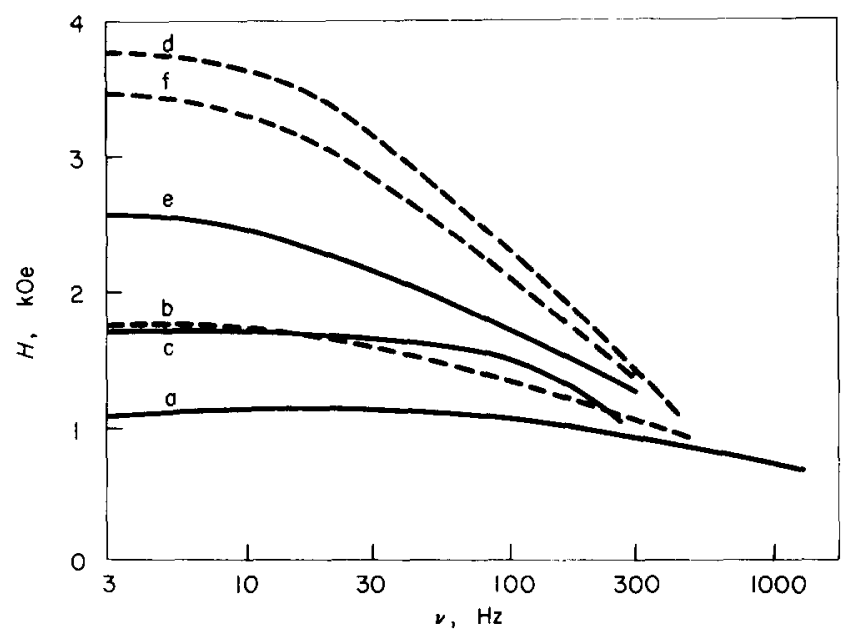

Fig. 5 Maximum amplitude of the field as a function of frequency and temperature for the coils indicated in Table 2

In Fig. 5 the maximum magnetic fields, which can be achieved with each of the three coil systems, are shown as a function of frequency. These magnetic fields are estimated from the currents measured in the secondary coils. At lower frequencies the larger primary coils are used for driving the systems.

The power supply used in these measurements was made with a RCA $2000 \mathrm{H}$ integrated circuit. 
Concerning the value of $I_{\max }$ we observed a phenomenon which has not yet been explained. When the coils are cooled down to liquid helium temperature for the second time, the values of $I_{\max }$ are reduced to about $90 \%$ of the original values. After warming up and cooling down again no further changes in these reduced values of $I_{\max }$ have been observed. The curves in the figures are related to these reduced values.

\section{Conclusion}

The experimental results, presented in this paper, show that the resonance method described provides a simple and cheap system for generating kilo-oersted ac magnetic fields. A disadvantage is that the capacitor in the resonance circuit should be changed in order to change the frequency and that, at very low frequencies, an enormous condensor would be required. In this case however the field coil can be connected directly to the power supply because the impedance of the field coil is sufficiently small at low frequencies. When a capacitor is used, the losses can be considerably reduced by placing the condensor in the helium bath.

We are very indebted to Prof W. Gröneveld of the Department of Electrical Engineering of our institute, for his valuable advice and stimulating interest in this work.

\section{References}

1 Brechna, H. Superconducting Magnet Systems (Springer-Verlag Berlin, 1973)

2 Penczynski, P., Hentzelt, H., Eger, G. Cryogenics 14 (1974) 503

3 Furtado, C.S. Cryogenics 12 (1972) 129

4 Van de Klundert, L.J.M., Gijsbertse, E.A., Van der Marel, L.C. Physica 69 (1973) 159

5 Kurtin, S., Foner, S., McNiff, E.J. Rev Sci Instr 38 (1967) 600

6 Kwasnitza, K., Horvath, I. Cryogenics 14 (1974) 71
Edited by

John A. G. Thomas
The 14 chapters of this book first appeared as papers in the journals Energy Policy and Food Policy. They form a snapshot of ongoing research, a record of the growing pains of a fledgling subject. The book reflects the debates and controversies about the strengths and weaknesses of energy analysis as an alternative to traditional economics for dealing with resource depletion problems.

contents

A review of methods - energy costs of fuels - energy costs of materials polymers and their alternatives - energy and food production - energy costs of goods and services - energy analysis of nuclear power stations - nuclear power and oil imports - energy analysis of power generating system - the economics of energy analysis - is net energy analysis any use? contributors

R. S. Atherton, Stephen Berry, Clark

Bullard, Peter Chapman, Michael Common,

Richard Denton, Robert Herendeen,

K. M. Hill, J. M. Holloman, Gerald Leach,

Thomas Long, Hiro Makino, David Pearce, B. Raz, M. Slesser, R. Treitel, F. J. Walford, Michael Webb and David Wright

ENERGY ANALYSIS is essential reading for planners in government and industry, policy analysts, statisticians, systems analysts, energy technologists, energy economists, environmentalists - in fact everyone concerned with the economics and planning of energy resources, production and consumption.

$275 \times 200 \mathrm{~mm} / 160$ pages / illustrations Cloth / 0902852604

Price $\mathbf{1 7 . 5 0}$ net $(\$ 20.00)$

\footnotetext{
$\begin{aligned} \text { ipc } & \text { Order your copy now from: } \\ \text { science } & \text { IPC Science and Technology Press Limited, }\end{aligned}$

and IPC House, 32 High Street, Guildford, Surrey, England GU1 3EW

technology Telephone: 0483-71661 Telex: Scitec Gd 859556
} 